\title{
Thermal Protection-, Aerodynamics- and Control Simulation of an Electromagnetically Launched Projectile
}

\author{
D. Lancelle ${ }^{1}$ and O. Božić ${ }^{1}$ \\ ${ }^{1}$ German Aerospace Center (DLR) - Inst. of Aerodynamics and Flow Technology, \\ Dept. Spacecraft, Braunschweig, Germany
}

\begin{abstract}
In recent years several ideas came up to apply electromagnetic launch technology for spaceflight applications. Using electric energy to propel a payload carrier promises the saving of propellant and therefore cost reduction for the transfer to orbit.

The conducted studies mostly comprise of a rough estimation of the launcher and the vehicle size. Sometimes a $\Delta v$-budget is given to illustrate the energy expenditure. Some of the studies neglect the necessity of a rocket engine. Only by means of an electromagnetic launch without the capability to maneuver, an orbit is not achievable.

The high acceleration and the high velocities at low altitude evoke high demands on the payload carrying vehicle. Its structure has to withstand the high acceleration forces during launch and the tremendous aerodynamic heat fluxes during the ascent flight in the dense atmosphere. Moreover a propulsion system, an attitude control system, and a flight controller are needed to bring the vehicle into a circular orbit.

This paper presents a vehicle concept that addresses all these demands. The vehicle comprises of a two stage hybrid rocket engine system, a thermal protection system, high test peroxide monopropellant thrusters for attitude control, and a guidance, navigation and control system. A simulation model is created, that consists of a 6-DOF flight mechanics module, aerodynamics model, propulsion module, thermal protection system simulation, as well as of guidance and flight control simulation. Therefore, the complete ascent with all its aspects can be simulated.

The simulation results show that a $710 \mathrm{~kg}$ vehicle launched with $2586 \mathrm{~g}$ and an initial velocity of $3642 \mathrm{~m} / \mathrm{s}$ can carry $31.5 \mathrm{~kg}$ of payload into a $300 \mathrm{~km}$ circular orbit. The configuration of the vehicle can be defined by a set of input parameters. This allows using the model within an optimization tool.
\end{abstract}

\section{ABBREVIATIONS}

CFD Computational Fluid Dynamics

DLR Deutsches Zentrum für Luft- und Raumfahrt

GNC Guidance Navigation and Control

IMU Inertial Motion Unit

LRA Lorentz Rail Accelerator

PEG Powered Explicit Guidance

PI Proportional-Integral

TPS Thermal Protection System

USAF United States Airforce

\section{INTRODUCTION}

There exist a number of studies related to the topic of using electromagnetic acceleration to launch payload into a low earth orbit. Several feasibility studies are carried out by the German Aerospace Center (DLR) within the last years [1], [2]. In these studies it is proposed to use a Lorentz accelerator (LRA, railgun) to accelerate a small vehicle with a total mass of roughly $500 \mathrm{~kg}$ to a start velocity of $3.3 \mathrm{~km} / \mathrm{s}$ with an acceleration of about $3300 \mathrm{~g}$. A rough description of the vehicle is given and the key parameters of the required railgun are derived in [3].

From this concept several problems arise. The vehicle has to withstand a high acceleration force, high aerothermal loads acting upon the vehicle's forebody, propulsion and an attitude control system is required and a fuel optimal ascent trajectory has to be considered. Furthermore, a more detailed layout of the vehicle has to be derived.

Problems concerning the high mechanical stress due to the high acceleration were discussed and solved in [4] and [5]. This paper deals with the remaining challenges and provides concepts to solve these problems. The different approaches for the specific problems are combined to a single computation model to simulate the ascent of an electromagnetically launched payload carrier.

\section{THERMAL PROTECTION SYSTEM}

To protect the vehicle from the intense aerothermal heat flux during the flight through the atmosphere, an ablative heat shield based on a carbon phenolic material is chosen, because of its simple setup; it does not need active components, pipes or cooling media. Such a thermal protection system is the best choice for short time heat protection. Moreover, carbon phenolic charring ablators are well approved in re-entry applications. 


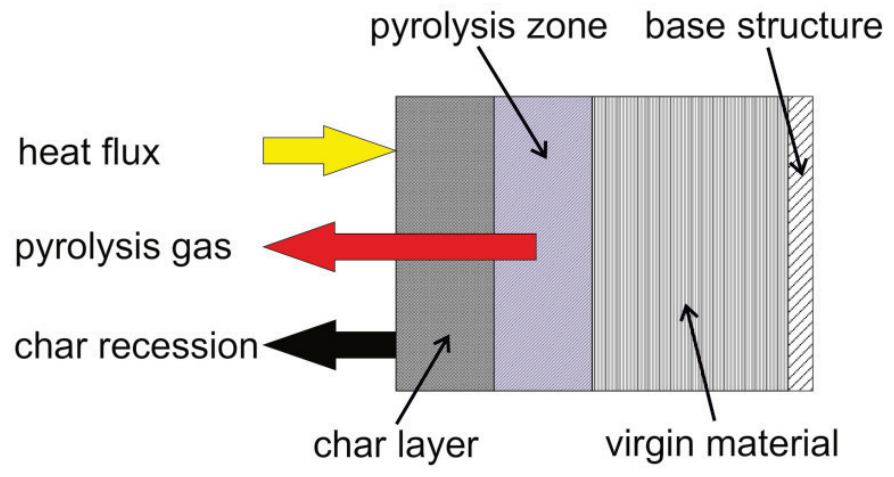

Fig. 1. Cross section of a charring ablative thermal protection system.

Fig. 1 shows the cross-section of a carbon phenolic heat shield. The ablator is a composite material made from carbon fibers embedded in a phenolic resin. The ablative material is mounted on a supporting base structure. The impinging heat flux heats up the material, until it undergoes a pyrolysis reaction. The virgin material is pyrolysed to carbon char. As the pyrolysis zone penetrates the material, a char layer is formed on top, which again is eroded due to oxidation and sublimation by the airflow. The gas produced during the pyrolysis reaction and the eroded char are injected into the boundary layer of the incoming flow. The pyrolysis reaction consumes energy and the mass injected into the boundary layer reduces the convective heat flux. These two mechanisms provide thermal protection.

To prove the feasibility of the application of an ablative heat shield for an electromagnetically launched payload vehicle, a software tool is created to simulate the TPS using a 1-D engineering approach for the ablator and the base structure. The tool includes the modelling of the pyrolysis reaction, the thermal conduction in the material and the base structure, the impinging heat flux, and the interaction of the injected material with the boundary layer. The model is implemented using different engineering tools and avoiding CFD-techniques, so that it can be used it within a multi-disciplinary-optimization.

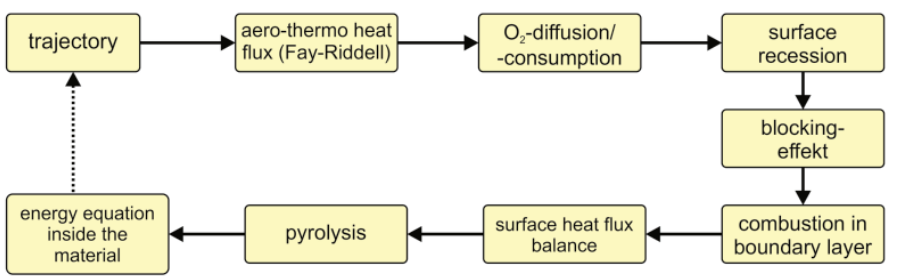

Fig. 2. Flowchart of the TPS model.

The computational sequence of one time step in the simulation is shown in Fig. 2. From the trajectory data, altitude and velocity are provided to the TPS-model. By means of the equations of Fay and Riddell [6] the convective cold wall heat flux to the stagnation point is determined. In the next step, the amount of oxygen, that reaches the surface of the heat shield by diffusion, is calculated. Then the surface recession and the decrease of convective heat flux, which is induced by the blowing of material into the boundary layer, are determined. In the next step, the influence of the combustion of char and pyrolysis gas within the boundary layer on the convective heat flux and oxygen consumption is calculated. Following, the surface heat flux balance is computed. With the net heat flux entering the ablator, the heat conduction and pyrolysis reaction can be derived. The program is capable of computing the change of the surface geometry, which again influences the aerodynamics. However, this was not used for the simulations presented in this paper.

\section{PROPULSION}

The vehicle cannot achieve an orbit around the earth without the use of any propulsion system. When it leaves the earth's atmosphere, its trajectory is only influenced by the gravity. The projectile then describes a keplerian orbit through the point where it leaves the atmosphere. This orbit is of elliptical shape and has its perigee below the earth's surface. Therefore a propulsion system is necessary to change the magnitude and direction of the velocity vector. Fig. 3 shows this circumstance.

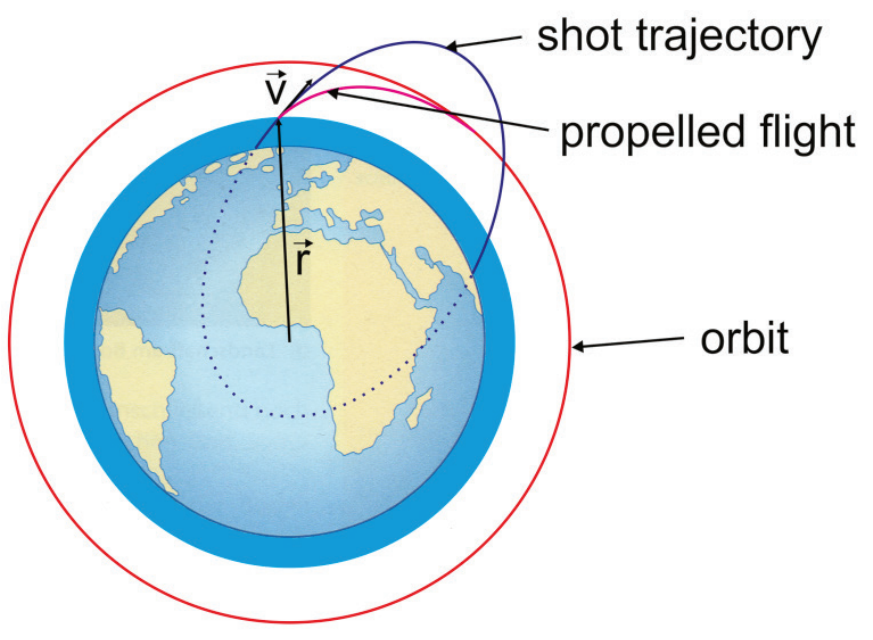

Fig. 3. Unpropelled and propelled flight trajectory of the vehicle.

A hybrid rocket engine is chosen as propulsion system, due to its promising performance capabilities and its safe handling. The engine uses hydroxyl-terminated polybutadiene with metallic additives as fuel and high concentrated hydrogen peroxide as oxidizer.

To verify the engine's concept and to assess its performance parameters, a test engine and a test bed are developed Fig. 4. The engine undergoes several tests and it is shown that the concept works very well. 


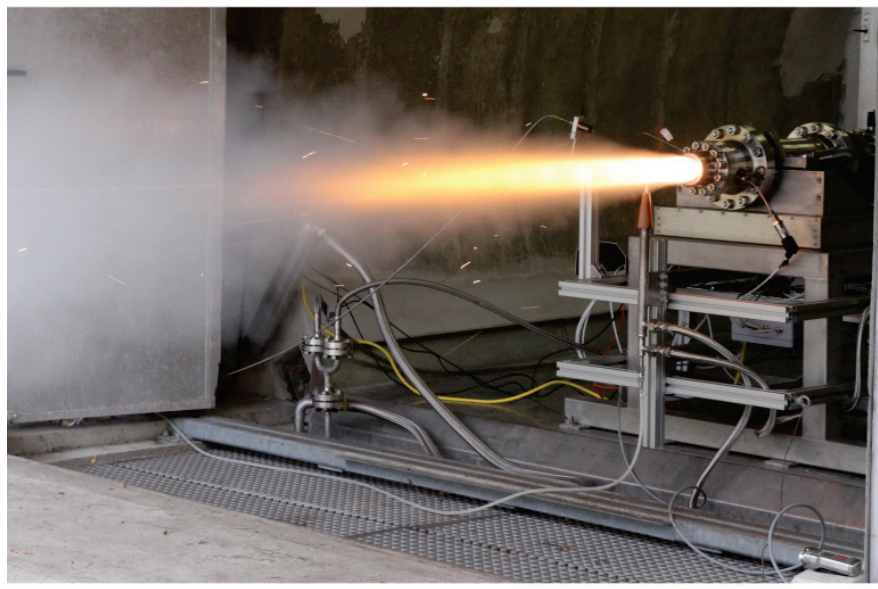

Fig. 4. Hybrid rocket engine test run.

\section{GUIDANCE NAVIGATION AND CONTROL}

To influence the flight characteristics of the projectile and to inject the vehicle into a circular orbit, a flight control system is necessary. The vehicle is controlled in the pitch and yaw axis by means of a reaction control system. It consists of four hydrogen peroxide monopropellant thrusters. Fig. 5 illustrates the pitch plane flight controller.

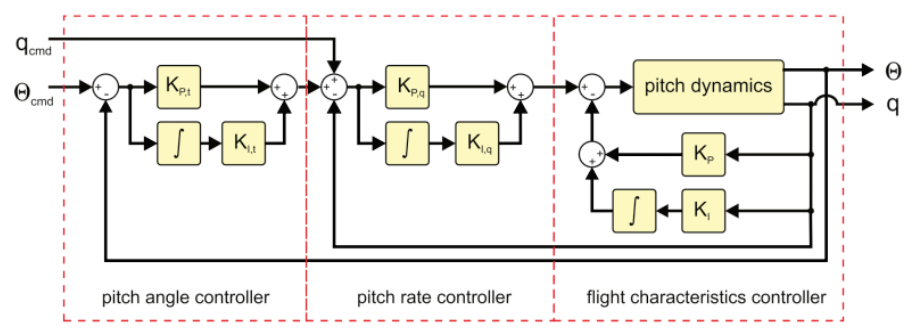

Fig. 5. Pitch plane flight controller.

To adjust the vehicles dynamic behavior, a flight characteristics controller is implemented as a PI-controller in the pitch rate feedback loop. The proportional gain provides damping, whereas the integral gain adjusts the stability. To inject the vehicle into a circular orbit, the flight controller needs to achieve commanded pitch angle and pitch rate values, provided by the guidance module. Therefore, a pitch rate and a pitch angle controller are implemented as a cascade control. Both controllers have a proportional and an integral part. To achieve the commanded values as fast as possible the commanded pitch rate is used for a reference-variable feed forward control. The flight controller of the yaw axis only consists of a flight characteristics controller.

The pitch program is generated by the guidance module. This module uses powered explicit guidance (PEG) with linear tangent steering. According to variation calculus, a propellant optimal trajectory into a circular orbit is achieved by linear variation of the tangent of the unit thrust vector direction $\underline{u}_{F}$ w.r.t. the inertial frame [7].

$$
\underline{u}_{F}=\frac{\underline{F}}{|\underline{F}|}=\frac{\underline{\lambda}_{F}}{\left|\underline{\lambda}_{F}\right|}=\frac{\underline{\lambda}_{v}+\dot{\dot{\lambda}}\left(t-t_{\lambda}\right)}{\sqrt{1+\dot{\lambda}^{2}\left(t-t_{\lambda}\right)^{2}}} .
$$

Equation (1) describes the time dependence of the direction of thrust [8]. $\underline{\lambda}_{F}$ is a vector representing the thrust direction. $\underline{\lambda}_{v}$ is a vector of unit length pointing in the direction of the velocity to be gained during the whole maneuver. $\underline{\dot{\lambda}}$ is a vector perpendicular to $\underline{\lambda}_{v}$ representing the rate of change of the thrust direction. The time $t_{\lambda}$ is chosen such that the total change in velocity generated by thrust is along $\underline{\lambda}_{v}[8]$.

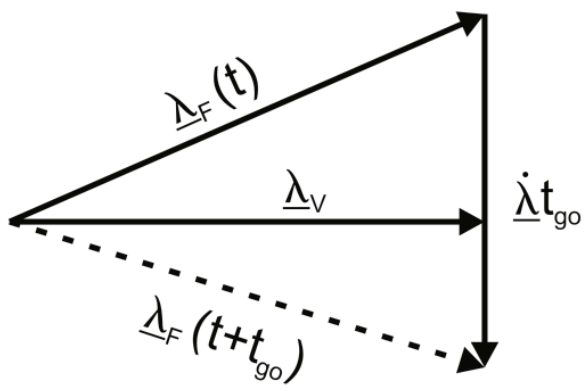

Fig. 6. Variation of thrust vector [8].

Fig. 6 illustrates the thrust vector direction according to equation (1). PEG starts at a specific time prior to first stage ignition. The guidance parameters $\underline{\lambda}_{v}, \underline{\dot{\lambda}}$, and $t_{\lambda}$ are calculated every 2 seconds, using a predictor-corrector scheme. $\underline{\lambda}_{v}$ is determined such, that the terminal velocity vector at orbit injection is achieved. $\dot{\lambda}$ is calculated to satisfy the terminal position constraints (orbit altitude).

\section{VEHICLE TOPOLOGY}

It is intended to simulate different vehicle configurations that shall be determined by a set of parameters. To create a complete vehicle based on a set of parameters, it is essential to define a basic topology. This is a basic concept that describes all the parts the vehicle incorporates and how they are arranged in relation to each other. The final dimensions of the components are derived from the parameters defining the vehicle and from pre-defined rules. The structural integrity of the vehicle is taken into account during this process. Finally, the total mass, the center of gravity and the moments of inertia are computed. Fig. 7 shows the topology of the vehicle. 


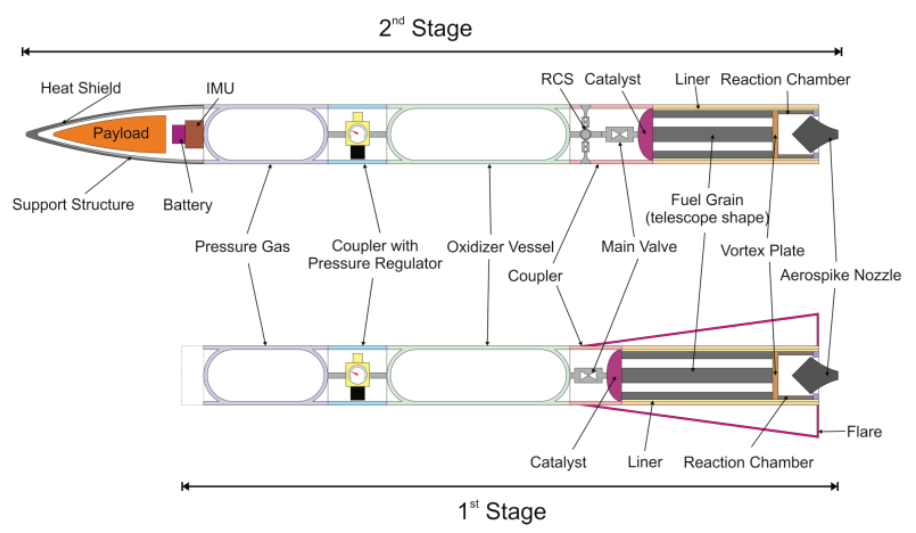

Fig. 7. Vehicle topology.

The vehicle design is carried out as a two stage hybrid rocket system. Each stage contains a pressure vessel for helium. This gas is needed to feed the oxidizer into the combustion chamber. The oxidizer is stored in pressure tanks as well. The two vessels are connected with a cylindrical coupler that houses a pressure regulator. The combustion chambers of both stages have the same setup. The solid fuel, which is carried out as a telescope fuel grain is housed in a liner for thermal insulation. At the back end of the fuel, a vortex plate is located to mix combustion products, so that they can fully react in the reaction chamber. For thrust generation, an aerospike nozzle is selected. This type of nozzle has an adaptive expansion and is more compact compared to a classical bell shape nozzle. Moreover it enables a higher expansion ratio and therefore a higher thrust coefficient. At the fuel grain inlet a catalyst bed is mounted. The catalyst decomposes the oxidizer to steam and oxygen at high temperatures, before it is injected into the combustion chamber. This eliminates the demand of an injector and ignition device. Oxidizer tank and combustion chamber are connected by a coupling device. This device also houses the engine's main valve. In contrast to the first stage, the second stage is equipped with a reaction control system for attitude control. The system is driven by catalytic decomposition of the oxidizer as a monopropellant.

The second stage is equipped with a nose cap with a powerlaw-shape. This shape is the best tradeoff between low aerodynamic drag and low aerothermal heating. The base structure is made from titanium. The nose cap is coated with an ablative thermal protection system. Beneath the nose cap the payload, the battery and an IMU with a flight computer are mounted. To provide flight stability, the first stage is equipped with a flare. Despite of the base structure of the nose, all structural parts are made from carbon fiber reinforced plastic.

\section{Simulation}

To simulate the ascent of an electromagnetically launched payload carrier in all its aspects, a sophisticated simulation tool is created. The computational models of the thermal protection system, guidance navigation and control module and vehicle parameterization are coupled to a 6-DOF flight mechanic simulation software. This software also incorporates a link to the USAF MISSILE DATCOM [9] software, to take aerodynamics into account. A model of the propulsion system is created as well. The composition of the simulation model is shown in Fig. 8 .

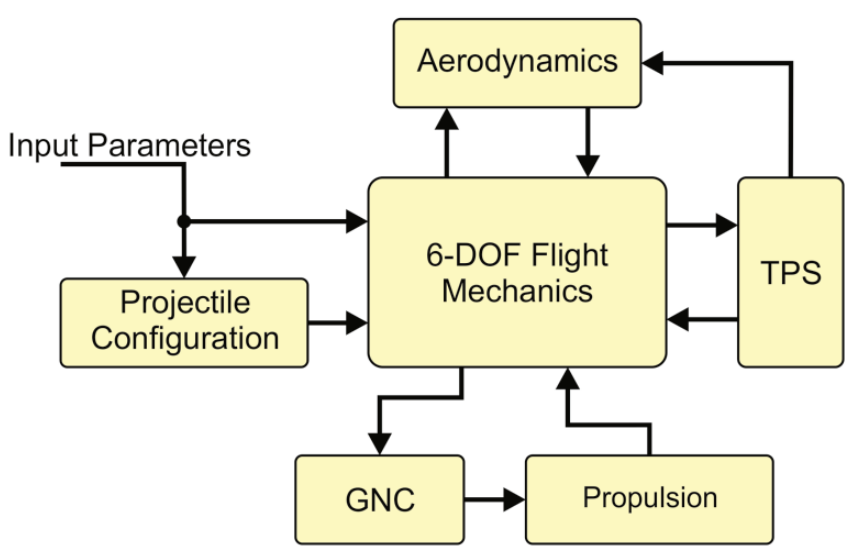

Fig. 8. Simulation model.

With the coupled software, the ascent of an electromagnetically launched vehicle, related to the feasibility studies described above is exemplarily simulated. The input parameters are listed in Table I.

\begin{tabular}{ll}
\hline Parameter & Value \\
\hline \hline total mass & $710 \mathrm{~kg}$ \\
payload mass & $31.5 \mathrm{~kg}$ \\
length & $6.87 \mathrm{~m}$ \\
diameter (w/o flare) & $0.37 \mathrm{~m}$ \\
TPS strength at stagnation point & $0.019 \mathrm{~m}$ \\
muzzle velocity & $3642 \mathrm{~m} / \mathrm{s}$ \\
launch angle & $43.7^{\circ}$ \\
\hline
\end{tabular}

Table I. Simulation parameters.

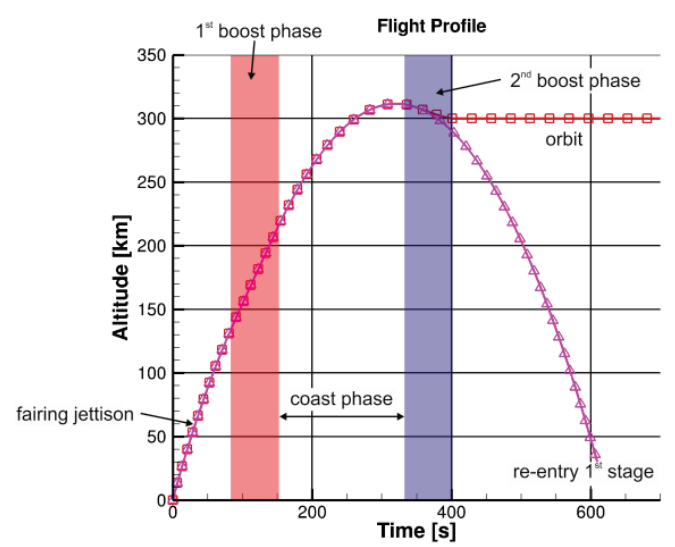

Fig. 9. Flight Profile. 
The computed flight profile is shown in Fig. 9. After the launch from the LRA, the projectile coasts up to $133 \mathrm{~km}$, where the first stage is ignited. Fairing jettison occurs en route at an altitude of about $60 \mathrm{~km}$, where aerodynamic drag and heat flux are sufficiently low. After burnout of the first stage, the vehicle undergoes an unpropelled coast phase. The apogee exceeds the orbit altitude for $10 \mathrm{~km}$. Next, the first stage is separated and the second stage is ignited to inject the vehicle into a circular orbit of $300 \mathrm{~km}$ of altitude.

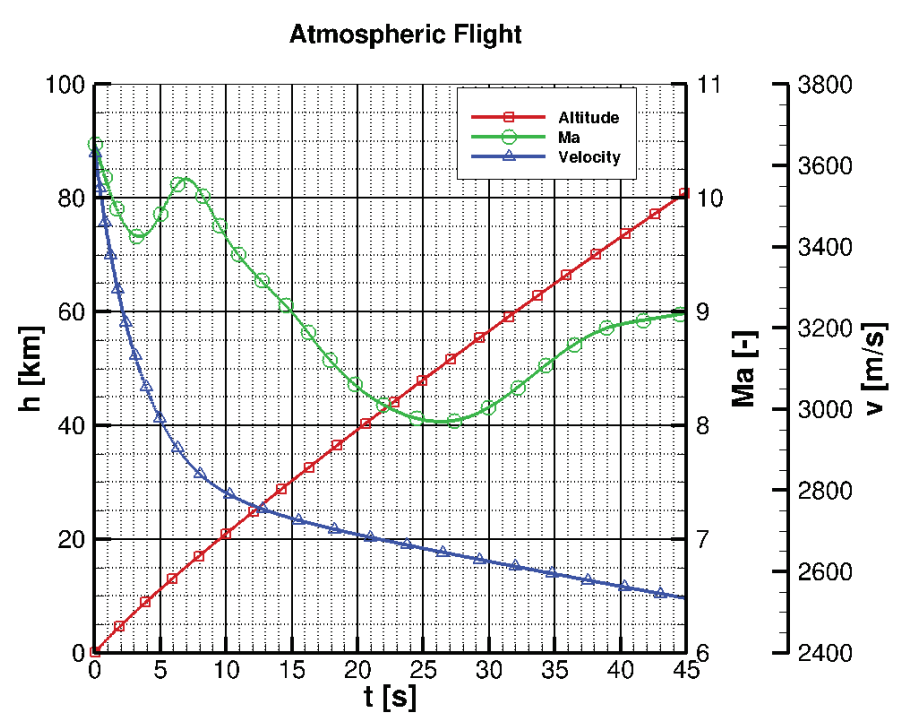

Fig. 10. Flight through lower atmosphere.

In Fig. 10 the first $45 \mathrm{~s}$ of flight until fairing jettison are shown. The flight velocity decreases strongly in the first $5 \mathrm{~s}$ of the flight. Thereafter the velocity slope is smaller. The Mach number is between 8.0 and 10.5 , depending on the altitude.

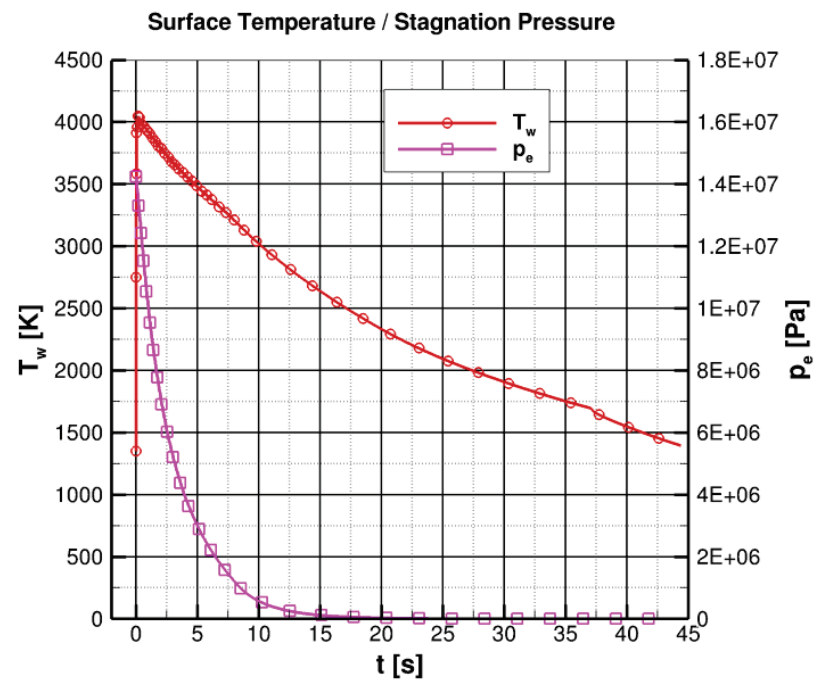

Fig. 11. Temperature and pressure at the stagnation point.
The surface temperature and pressure in the stagnation region for bluntness radius of $9.7 \mathrm{~mm}$ are shown in Fig. 11. At launch, where the highest velocity and the highest atmospheric density exist, the stagnation pressure and the surface temperature have their maximum value. With increasing altitude and decreasing velocity, the pressure and temperature decrease as well. The peak surface temperature is about $4050 \mathrm{~K}$, whereas the peak pressure has a value about 142 bar. The peak heat flux was determined with $8.3 \times 10^{7} \mathrm{~W} / \mathrm{m}^{2}$.

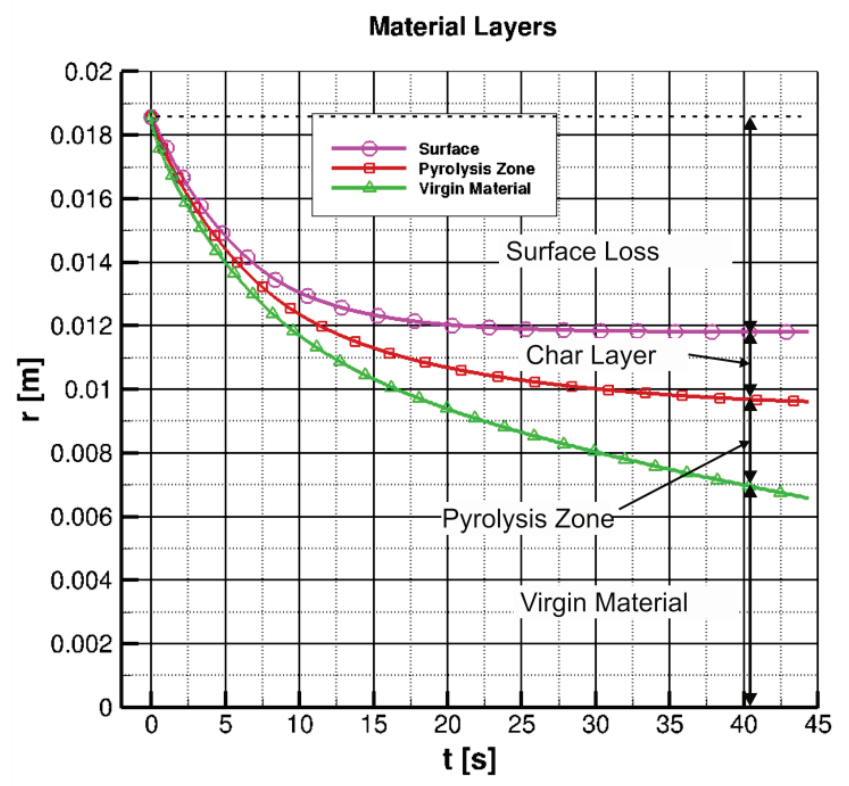

Fig. 12. Material layer evolution.

Fig. 12 shows the layer evolution in the cross section of the material at the stagnation point. At the beginning of the flight, the char layer and the pyrolysis zone are consumed almost instantly. This is due to the high heat flux and stagnation pressure. The surface recession and pyrolysis reaction decreases over the flight time with increasing altitude, and a char layer and a pyrolysis zone are formed. The surface recession stops at about 30 seconds. The char layer and pyrolysis zone further penetrate the material due to distribution of latent heat. The total surface loss is observed as $6.8 \mathrm{~mm}$, whereas the char layer has a thickness of roughly $2.2 \mathrm{~mm}$. At fairing jettison, the remaining virgin material has a thickness of $6.6 \mathrm{~mm}$.

Fig. 13 displays the temperature distribution in the material cross section at the stagnation point. A high temperature gradient is observed in the first seconds of the flight, moving deeper into the material with increasing surface recession. The temperature gradient is decreasing with decreasing heat load and the latent thermal energy distributes in the material. The structure material does not heat up significantly, as the vehicle is exposed to the high heat loads only for a short period. Thus a good thermal protection is provided and the vehicle is capable to withstand the high thermal loads. 


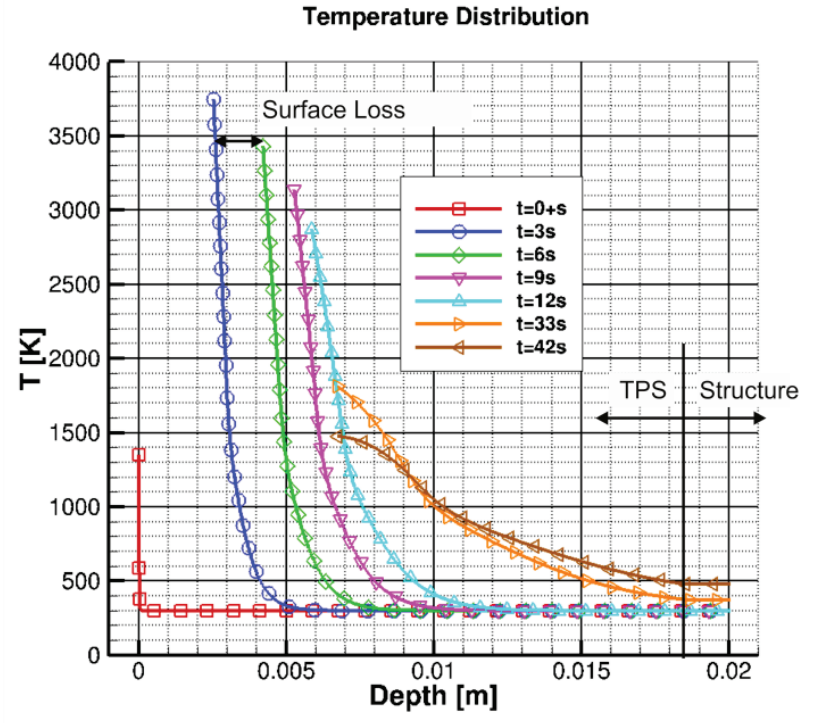

Fig. 13. Temperature distribution.

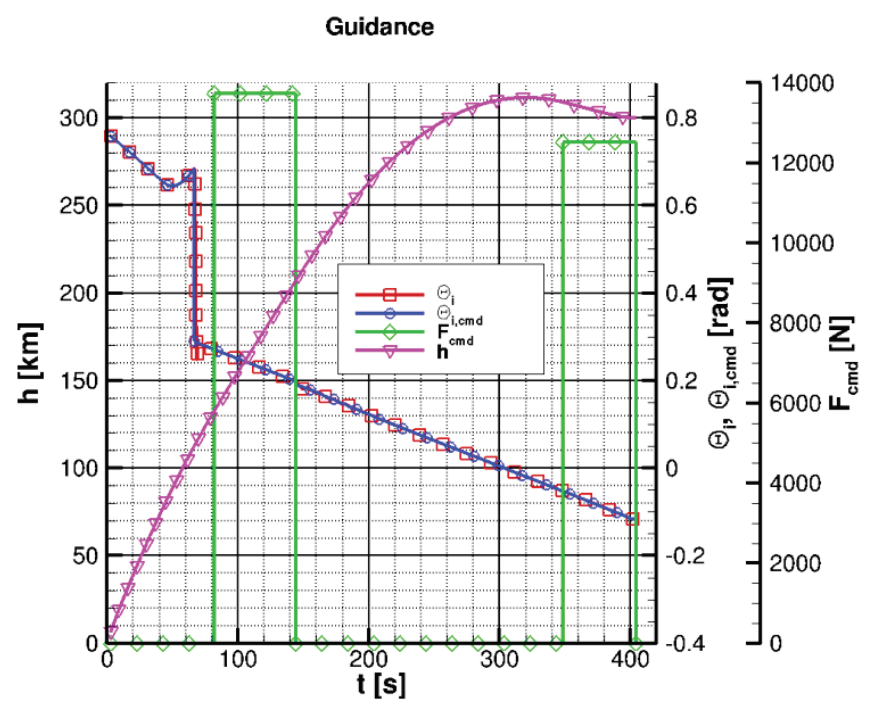

Fig. 14. Linear tangent steering to orbit.

Fig. 14 shows the linear tangent steering behavior of the vehicle during the ascent to orbit. It can be observed, that the flight controller fulfills the commands from the guidance module very well; the pitch angle in the inertial space follows the commanded pitch angle with very small deviation. One can see the linear variation of the tangent of the pitch angle over the maneuver time. The guidance algorithm is started a specific period before the ignition of the first stage. Thus, the vehicle has the correct attitude at the begin of the first boost phase.

\section{SUMMARY}

Based on feasibility studies, a detailed concept of an electromagnetically launched payload vehicle for a low earth orbit is elaborated. The main problems arising from such a concept, like high acceleration forces, aerothermal heating, and the necessity of a propulsion system and attitude control are identified and solutions are provided. A computation model is created to simulate the ascent of the vehicle in all its aspects. The simulation results underline the feasibility of the concept. It is shown that a vehicle with a mass of $710 \mathrm{~kg}$ can bring $31.5 \mathrm{~kg}$ payload into orbit. The vehicle setup is determined by a set of parameters. The complete model is based on engineering tools, which save computational power. Thus, an optimization model can be used to determine an optimal vehicle configuration to provide low specific payload cost for the transfer to a low earth orbit. Such an optimization is currently in progress.

\section{ACKNOWLEDGMENT}

The authors would like to thank Dr.-Ing. Michael Gräßlin, with Steinbeis-Transferzentrum Raumfahrt, for the fruitful discussion about powered explicit guidance.

\section{REFERENCES}

[1] O. Božić, T. Eggers, S. Wiggen, "Aerothermal and Flight Mechanic Considerations by Development of Small Launchers for Low Orbit Payloads Started from Lorentz Rail Accelerator," 3rd European Conference for Aero-Space Sciences - EUCASS, 6-9 July 2009, Versailles-Paris, France, Progress in Propulsion Physics, Vol. 2, (edited by L. DeLuca, C. Bonal, O. Heidn, S. Frolov,), EUCASS advances in aerospace sciences book series, EDP sciences, pp. 765-784, Torus Press, 2011

[2] O. Božić, D. Porrmann, M. Schneider, "Conceptual Design of "SILVER EAGLE" - combined Electromagnetic and Hybrid Rocket System for Suborbital Investigations", 61st International Astronautical Congress (IAC), 27th Sept. - 1st Oct. 2010, C4.6. Special Session on "Missions Enabled by New Propulsion Technologies and Systems", IAC-10-C6.4.1, Prague, Czech Republic

[3] M. Schneider, O. Božić, T. Eggers, "Some Aspects concerning the Design of Multistage Earth Orbit Launchers using Electromagnetic Acceleration", 15th Electromagnetic Launch Symposium, May 17 - 20, 2010, Brussels, Belgium, IEEE Transaction of Plasma Science, Vol. 39, Nr. 1, Part II, Jan. 2011, ISSN 0093-3813

[4] D. Lancelle, O. Božić, J. Martinez-Schramm, S. Linke, "Measurement of Mechanical Properties of Solid Fuels and Advanced Composite Materials related to Short-Time High Acceleration generated by Lorentz Rail Accelerators", 15th Electromagnetic Launch Symposium, May 17 - 20, 2010, Brussels, Belgium, IEEE Transaction of Plasma Science, Vol. 39, Nr. 1, Part I, Jan. 2011, ISSN 0093-3813, TPS 3849

[5] D. Lancelle, O. Božić, H. Köke, "Flight Test Results of the Investigation of Acceleration Effects on a Gun-Launched Rocket Engine", IEEE Transactions on Plasma Science, Vol. 41, No. 5, Part I, ISSN 0093-3813, 2013

[6] J. A. Fay, F. R. Riddell, "Theory of Stagnation Point Heat Transfer in Dissociated Air", Journal of Aeronautical Sciences, Vol. 25, No. 2, pp. $73-$ $85,121,1958$

[7] D. F. Lawden: "Optimal Trajectories for Space Navigation", Butterworths, London, 1963

[8] R. L. McHenry, et. al., "Space Shuttle Ascent Guidance, Navigation and Control", The Journal of the Astronautical Sciences, Vol. XXVII, No. 1, 1979

[9] S. R. Vukelich, S. L. Stoy, K. A. Burns, "MISSILE DATCON, Volume IFinal Report", Flight Dynamic Laboratory, Air Force Wright Aeronautical Laboratories, AFWAL-TR-86-3091, Ohio, 1988 